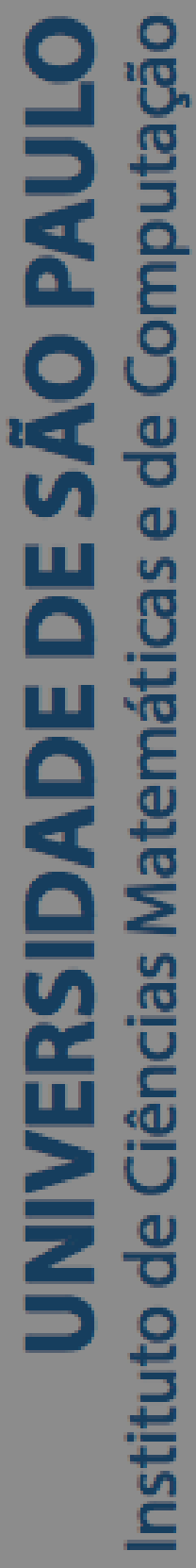

\title{
Percolação Acessível
}

\section{Ricardo de Jesus Caldas Assis}

Dissertação de Mestrado do Programa Interinstitucional de Pós-

Graduação em Estatística (PIPGEs) 

Data de Depósito:

Assinatura:

Ricardo de Jesus Caldas Assis

\title{
Percolação Acessível
}

\begin{abstract}
Dissertação apresentada ao Instituto de Ciências Matemáticas e de Computação - ICMC-USP e ao Departamento de Estatística - DEs-UFSCar, como parte dos requisitos para obtenção do título de Mestre em Estatística - Programa Interinstitucional de Pós-Graduação em Estatística. VERSÃO REVISADA Área de Concentração: Estatística

Orientador: Prof. Dr. Pablo Martín Rodriguez
\end{abstract}


Ficha catalográfica elaborada pela Biblioteca Prof. Achille Bassi e Seção Técnica de Informática, ICMC/USP, com os dados inseridos pelo(a) autor(a)

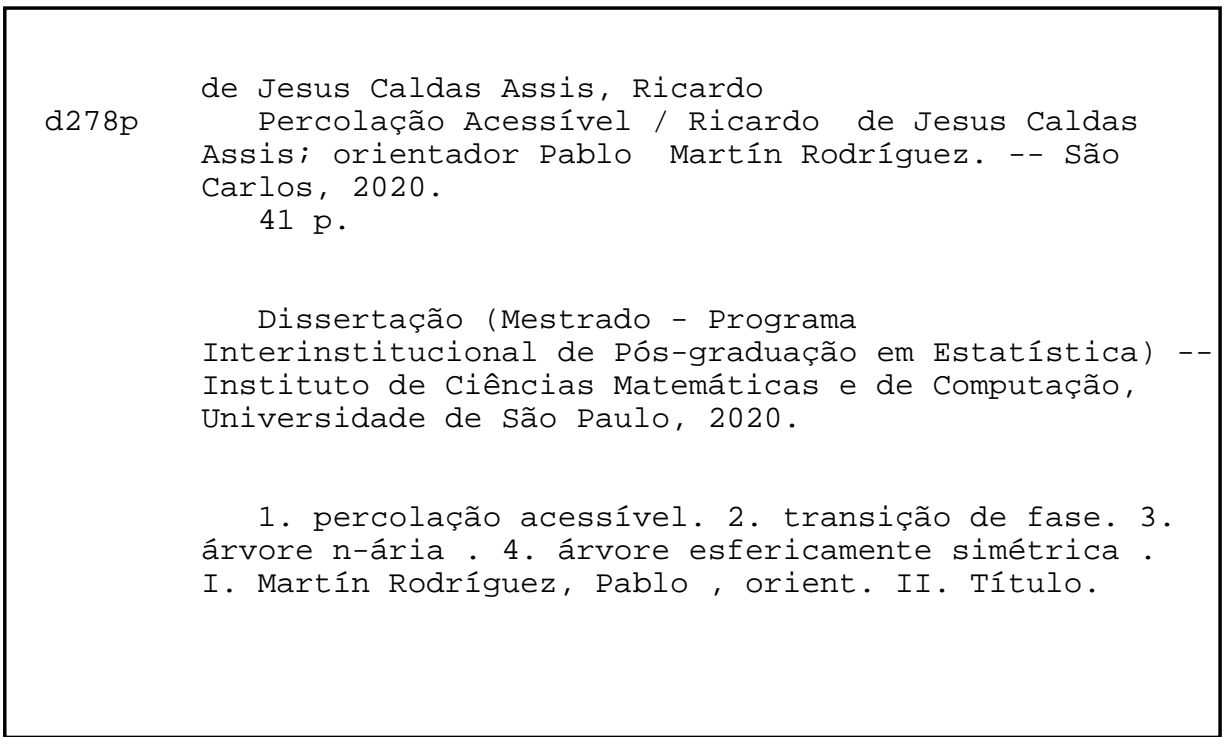

Bibliotecários responsáveis pela estrutura de catalogação da publicação de acordo com a AACR2: Gláucia Maria Saia Cristianini - CRB - 8/4938 Juliana de Souza Moraes - CRB - 8/6176 


\section{Ricardo de Jesus Caldas Assis}

\section{Accessible Percolation}

Master dissertation submitted to the Institute of Mathematics and Computer Sciences - ICMC- USP and to the Department of Statistics - DEs- UFSCar, in partial fulfillment of the requirements for the degree of the Master Interagency Program Graduate in Statistics. FINAL VERSION

Concentration Area: Statistics

Advisor: Prof. Dr. Pablo Martín Rodriguez

USP - São Carlos

April 2020 


\section{UNIVERSIDADE FEDERAL DE SÃO CARLOS}

Centro de Ciências Exatas e de Tecnologia

Programa Interinstitucional de Pós-Graduação em Estatística

\section{Folha de Aprovação}

Assinaturas dos membros da comissão examinadora que avaliou e aprovou a Defesa de Dissertação de Mestrado do candidato Ricardo de Jesus Caldas Assis, realizada em 17/02/2020:

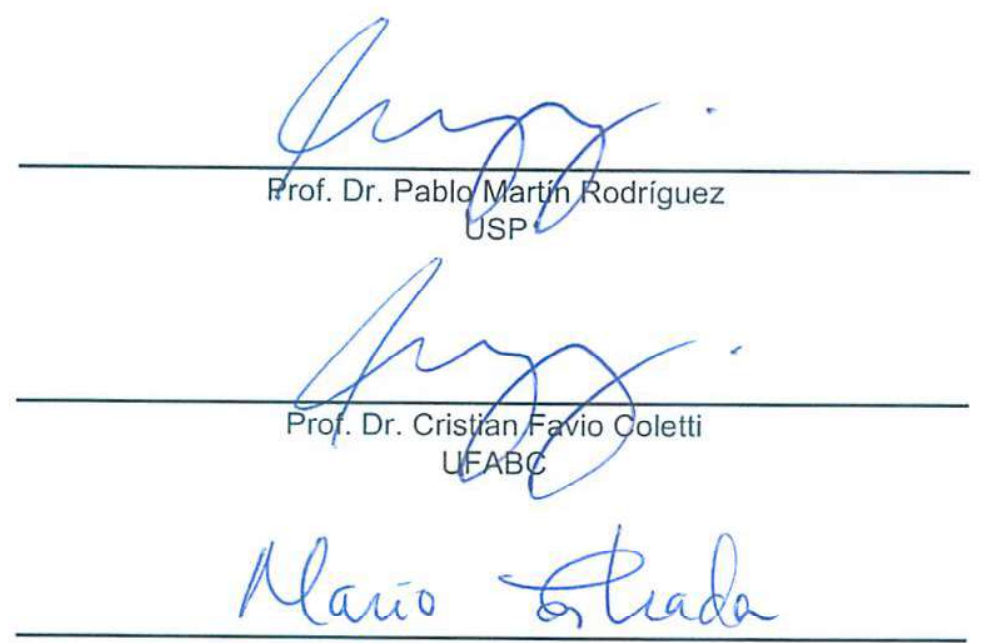

Prof. Dr. Mário Andrés Estrada López

UFPE

Certifico que a defesa realizou-se com a participação à distância do(s) membro(s) Cristian Favio Coletti e, depois das arguições e deliberações realizadas, o(s) participante(s) à distância está(ao) de acordo com o conteúdo do parecer da banca examinadora redigido neste relatório de defesa.

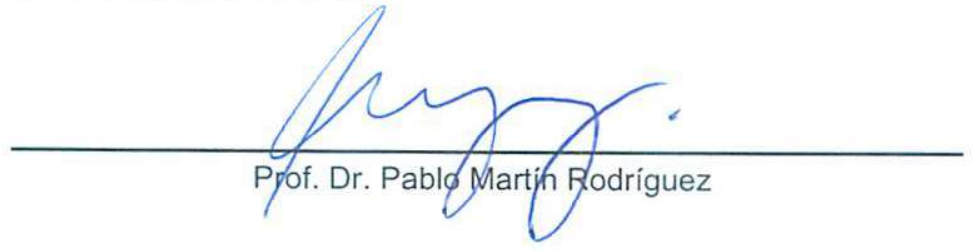





\section{Resumo}

ASSIS, R. J. C. Percolação Acessível. 2020. 41p. Dissertação (Mestrado em Estatística

- Programa Interinstitucional de Pós-Graduação em Estatítica) - Instituto de Ciências Matemáticas e de Computação, Universidade de São Paulo, São Carlos - SP, 2020.

Consideramos o modelo de percolação acessível na árvore $n$-ária finita de altura $h$. O modelo é definido associando-se uma variável aleatória contínua $X_{v}$ para cada vértice $v$ da árvore. A principal questão a ser considerada e estudada é a existência ou não de um caminho de vizinhos mais próximos $v_{0}, v_{1}, \ldots, v_{n}$, conectando a raiz com a fronteira da árvore, de tal modo que $X_{v_{0}}<X_{v_{1}}<\ldots<X_{v_{n}}$. O evento definido pela existência desse caminho é chamado de percolação acessível. Neste trabalho estudamos a probabilidade do evento de percolação acessível quando o valor $n$ é dado por $n(h)=\alpha(h) h$ em que $h$ é a altura da árvore e $\alpha(h)$ é constante. Os resultados são obtidos fazendo $h \rightarrow \infty$. Adicionalmente, discutiremos outros resultados recentes na literatura.

Palavras chave: Transição de Fase, Percolação Acessível, Árvore $n$-ária 


\section{Abstract}

ASSIS, R. J. C. Accessible Percolation. 2020. 41p. Dissertação (Mestrado em Estatística - Programa Interinstitucional de Pós-Graduação em Estatítica) - Instituto de Ciências Matemáticas e de Computação, Universidade de São Paulo, São Carlos - SP, 2020 .

We consider the accessibility percolation model on the $n$-ary tree height the finite $h$. The model is defined by associating a continuous random variable $X_{v}$ for each vertex $v$ in the tree. The main issue to consider and study is whether or not there is a nearest neighbor path $v_{0}, v_{1}, \ldots, v_{n}$, connecting the root to the tree boundary, such that $X_{v_{0}}<$ $X_{v_{1}}<\ldots<X_{v_{n}}$. The event defined by the existence of this path is called the accessible percolation. In this paper we study the probability of the accessible percolation event. when $n$ is given by $n(h)=\alpha(h) h$ where $h$ is the height of the tree and $\alpha(h)$ is constant. Results are obtained by making $h \rightarrow \infty$. Additionally, we will discuss other recent results in the literature.

keywords: Phase Transition, Accessible Percolation, $n$-ary Tree 


\section{Lista de Figuras}

1.1 grafo orientado com laço . . . . . . . . . . . . . . . . 4

1.2 árvore dos vértices numerados de 1 a $15 \ldots \ldots \ldots \ldots$

1.3 árvore dos vértices numerados de 1 a $12 \ldots \ldots \ldots$

1.4 2-árvore de raiz $\omega \ldots \ldots \ldots \ldots$

1.5 árvore esféricamente simétrica $T \ldots \ldots \ldots \ldots$

1.6 Uma representação da árvore $T_{!}$, até o nível $i=4 \ldots \ldots$. . . . . . . . 10

2.1 realizacão de um modelo de percolação acessível na 2-árvore $T$. . . . . . . 12

2.2 A correlação entre dois caminhos depende apenas do número $h-k+1$ de vértices que ambos os caminhos têm em comum. . . . . . . . . . . . . 15

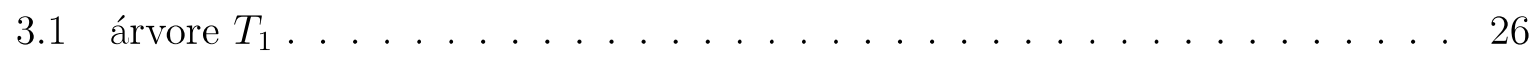

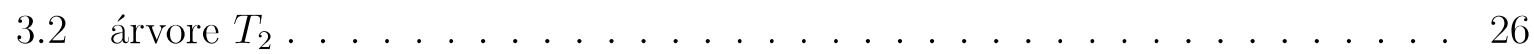





\section{Sumário}

$\begin{array}{ll}\text { Introdução } & 1\end{array}$

1 Preliminares e notação de grafos 3

2 Modelo de percolação acessível em árvores $n$-árias $\quad 11$

2.1 O modelo . . . . . . . . . . . . . . . . . . . . . . 11

2.2 Número de caminhos acessíveis . . . . . . . . . . . . . . . . . . . 13

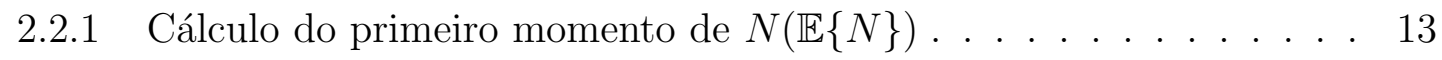

2.2.2 Cálculo do segundo momento de $N \ldots \ldots$. . . . . . . 14

2.3 Probabilidade de existir caminhos acessíveis na $n$-árvore $\ldots \ldots \ldots$

3 Discussão $\quad 24$

3.1 Discussão . . . . . . . . . . . . . . . . . . . . 24

$\begin{array}{ll}\text { Conclusão } & 29\end{array}$ 


\section{Introdução}

O modelo de percolação acessível em árvores foi introduzido no artigo [1], inspirado em questões de Biologia Evolutiva (A questão principal é supor uma população de alguma forma de vida que tenha o mesmo tipo genético (genótipo). Se ocorrer uma mutação, é criado um novo genótipo que pode morrer ou substituir o antigo. Desde que a seleção natural seja suficientemente forte, o último só acontece se o novo genótipo tiver maior aptidão). Nesse artigo uma $n$-árvore de altura $h$ é considerada e uma variável aleatória absolutamente contínua $X_{v}$ é dada e está associada a cada vértice $v$, independentemente de todo o resto. Uma das principais questões neste modelo é se existe um caminho de vizinhos mais próximos $v_{1}, v_{2}, v_{3}, \ldots, v_{h}$ de tal modo que $X_{v_{1}}<X_{v_{2}}<X_{v_{3}}<\ldots<X_{v_{h}}$. Esse tipo de caminho é chamado de caminho acessível. No artigo [1] os autores derivaram um resultado assintótico para a probabilidade de ter pelo menos um caminho acessível conectando a raiz com o nível $h$ de uma árvore $n(h)$ com $n(h):=\alpha(h) h$, e $\alpha$ alguma constante positiva arbitrária. Consequentemente, eles provaram a existência de um valor crítico de percolação para este modelo quando $h \rightarrow \infty$. De fato, eles mostraram que a probabilidade de ter pelo menos um caminho acessível vai para zero para $\alpha<\alpha_{c}$ e converge para um número positivo para $\alpha>\alpha_{c}$ com $1 / e \leq \alpha_{c} \leq 1$, onde $\alpha_{c}$ é o valor crítico de percolação. Mais tarde, este resultado foi complementado no artigo [2], onde os autores mostraram que essa probabilidade converge para 1 quando $\alpha>1 / e$. Recentemente, um problema relacionado foi analisado no hipercubo $H_{N}=\{0,1\}^{N}$, com $N \in \mathbb{N}$, para mais confira os artigos [4,6]. Sugerimos consultar tambémos artigos $[8,9,10,11,12]$ para questões relacionadas. No artigo [3], a principal questão estudada é a existência de um caminho acessível infinito na árvore esfericamente simétrica. Neste modelo para cada vértice $v$ de uma árvore esfericamente simétrica associa-se uma variável aleatória absolutamente contínua $X_{v}$ com distribuição uniforme $(0,1)$. Prova-se que para uma dada função de crescimento da árvore existe um valor crítico de percolação. 
Nesta dissertação vamos descrever os argumentos usados em [1] para a prova de transição de fase do modelo. Além disto, vamos discutir problemas relacionados e problemas em aberto. O capítulo 1 é dedicado para estudarmos um pouco de teoria de grafos (definição, exemplos e propriedades) e conceituarmos árvores e algumas de suas principais propriedades. No capítulo 2 foi feito um estudo sistemático do artigo [1]. Por fim, no capítulo 3 foi feita uma discussão sobre [3]. 


\section{Capítulo 1}

\section{Preliminares e notação de grafos}

Neste capítulo veremos o que é um grafo e algumas definições relacionadas a grafos. Também veremos o conceito de $n$-árvore ou árvore $n$-ária e árvore esfericamente simétrica.

Definição 1.1 (Cormen, Leiserson, Rivest, Stein. (2009)). Um grafo orientado finito $G$ é uma par $G=(V, E)$, onde

1. V é um conjunto finito, chamado de conjunto de vértices;

2. E é uma relação binária em $V$, chamado de conjunto de arestas.

Em um grafo não orientado $G=(V, E), E$ consiste de pares de vértices não ordenados. Se $E$ é infinito, dizemos que $G$ é um grafo infinito.

Observação 1.1. Os grafos podem ser representados graficamente: Os vértices são desenhados como círculos e as arestas são desenhadas como curvas (retas ou não) ligando dois círculos, no caso de grafos orientados, as curvas tem um seta em uma das extremidades. 
Exemplo 1.1. O grafo a seguir é um exemplo de grafo orientado com laço (arestas de um vértice para ele mesmo).

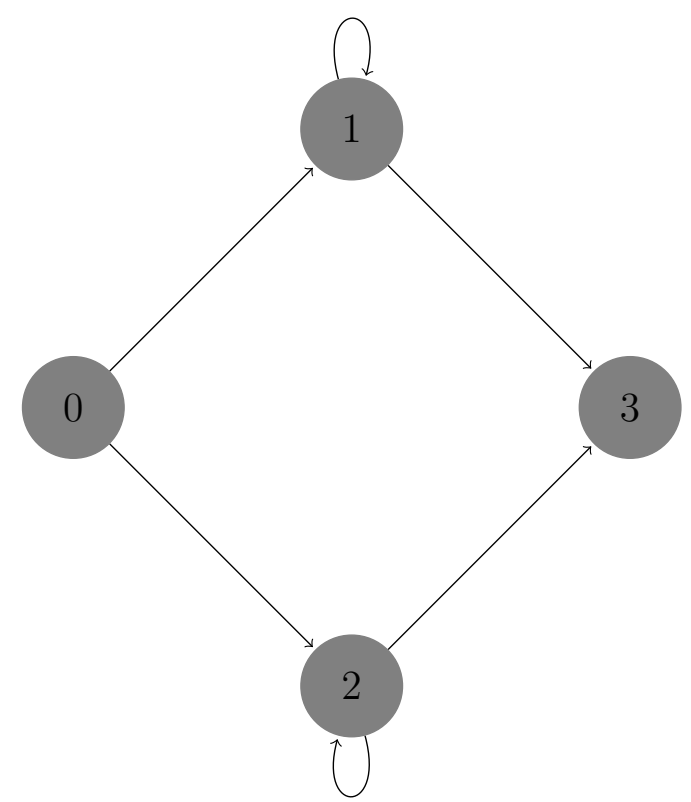

Figura 1.1: grafo orientado com laço

Para este grafo, temos os seguintes conjuntos de vértices e arestas

$$
V=\{0,1,2,3\} \text { e } E=\{(0,1),(0,2),(1,1),(1,3),(2,2),(2,3)\}
$$

Seja $G=(V, E)$ um grafo. Um caminho de comprimento $k$ de um vértice $u$ até um vértice $u^{\prime}$ em $G$ é uma sequência $v_{0}, v_{1}, v_{2}, \ldots, v_{k}$ de vértices tal que $u=v_{0}, u^{\prime}=$ $v_{k}$ e $\left(v_{i-1}, v_{i}\right) \in E$ para $i \in\{1,2, \ldots, k\}$. Além disso, dizemos que um caminho é simples se todos os vértices no caminho são distintos. Por outro lado, um caminho $v_{0}, v_{1}, \ldots, v_{k}$ forma um ciclo se $v_{0}=v_{k}$ e o caminho contém pelo menos uma aresta. O ciclo é simples se além disso $v_{1}, v_{2}, \ldots, v_{k}$ são distintos. Agora, se $G$ é sem ciclo, então ele é dito ser acíclico. Finalmente, $G$ é conexo se cada vértice é acessível a partir de todos os outros. Agora, dizemos que se $G$ é conexo acíclico, então $G$ é uma árvore. Em outras palavras, uma árvore $T$ é um grafo tal que todo par de vértices de $T$ é unido por um único caminho.

Observação 1.2. Dizemos que uma árvore $T$ é infinita se $V$ e E são infinitos. Mais ainda, se $T$ é infinita, então dizemos que $T$ é localmente finita, se cada vértice $v$ da árvore tem um número finito de vizinhos mais próximos em $T$. 
Exemplo 1.2. A figura abaixo é uma ilustação de uma árvore não-orientada.

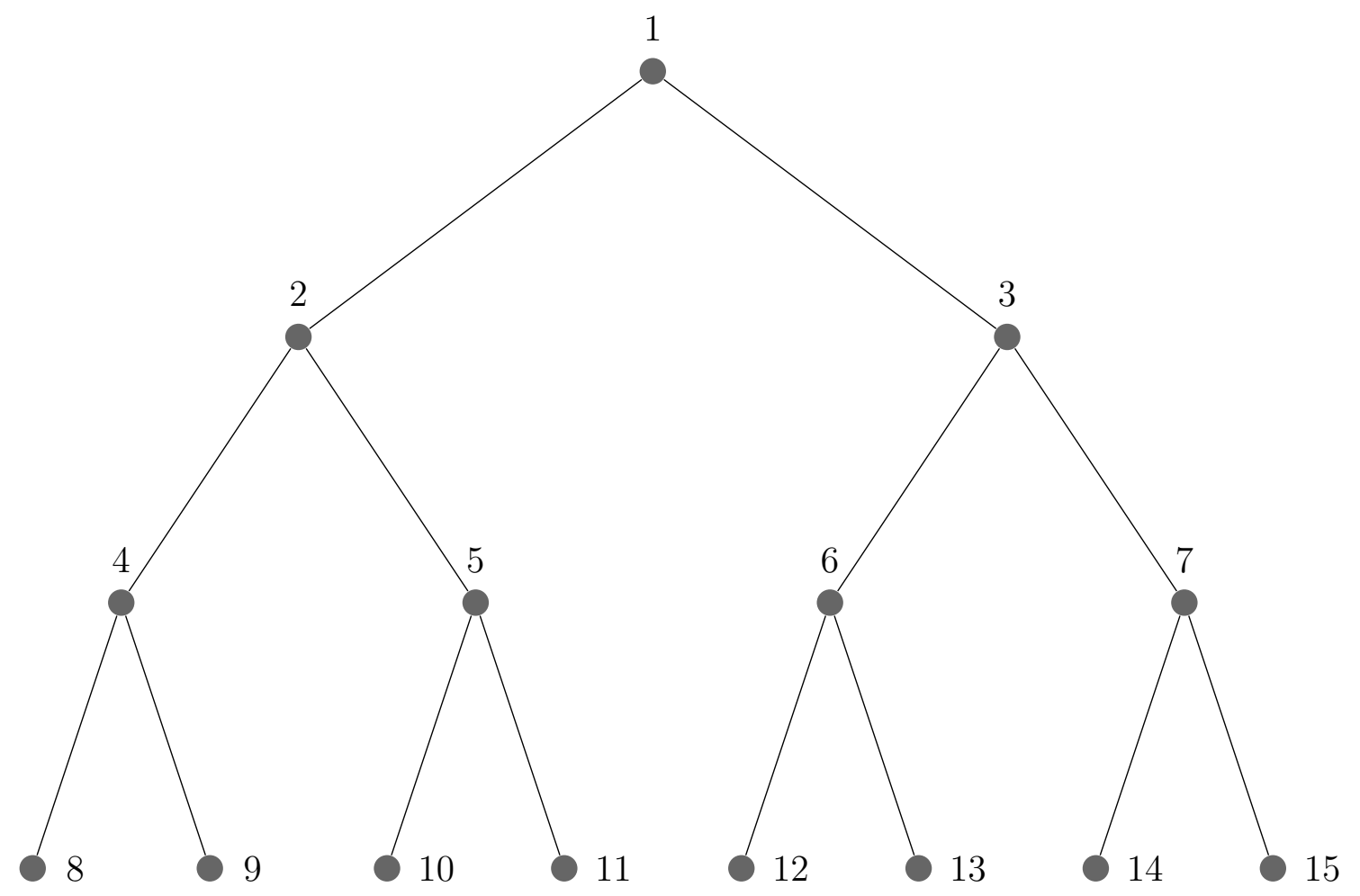

Figura 1.2: árvore dos vértices numerados de 1 a 15

Já vimos que uma árvore é um grafo acíclico conexo não orientado. Agora, uma árvore com raiz é uma árvore que tem um vértice de origem denotado por $\mathbf{0 .}$

Exemplo 1.3. O grafo da Figura 1.2 é uma árvore com origem (raiz) exatamente no vétice de número 1.

As definições que seguem valem para quaisquer grafos. Entretanto, vamos restringilas a árvores.

Definição 1.2. Seja $T=(\vartheta, \xi)$ uma árvore. Um caminho de tamanho $n$ em $T$ é uma sequência de vértices $v_{0}, v_{1}, v_{2}, \ldots, v_{n}$ conectados por uma sequência de elos $\varepsilon_{1}, \varepsilon_{2}, \ldots, \varepsilon_{n}$, tais que $\varepsilon_{i}=\left(v_{i-1}, v_{i}\right)$ para todo $i \in\{1,2, \ldots, n\}$. Denotamos esse caminho por $v_{0} \rightarrow v_{n}$.

Observação 1.3. No caso da definição anterior, dizemos que a distância de $v_{0}$ até $v_{n}$ é $n$ e denotamos por $d\left(v_{0}, v_{n}\right)=n$.

Definição 1.3. Seja $T=(\vartheta, \xi)$ uma árvore. Dizemos que $u$ e $v$ de $\vartheta$ são vizinhos mais próximos se $d(u, v)=1$. 
Definição 1.4. Seja $T=(\vartheta, \xi)$ uma árvore. Dizemos que o grau de $v$, denotado por $g(v)$, é o número de vizinhos mais próximos de $v$.

Observação 1.4. Considere o conjunto $G_{v}=\{u \in \vartheta ; d(v, u)=1\}$. Então, $g(v)=\left|G_{v}\right|$.

Exemplo 1.4. Seja a árvore finita, abaixo.

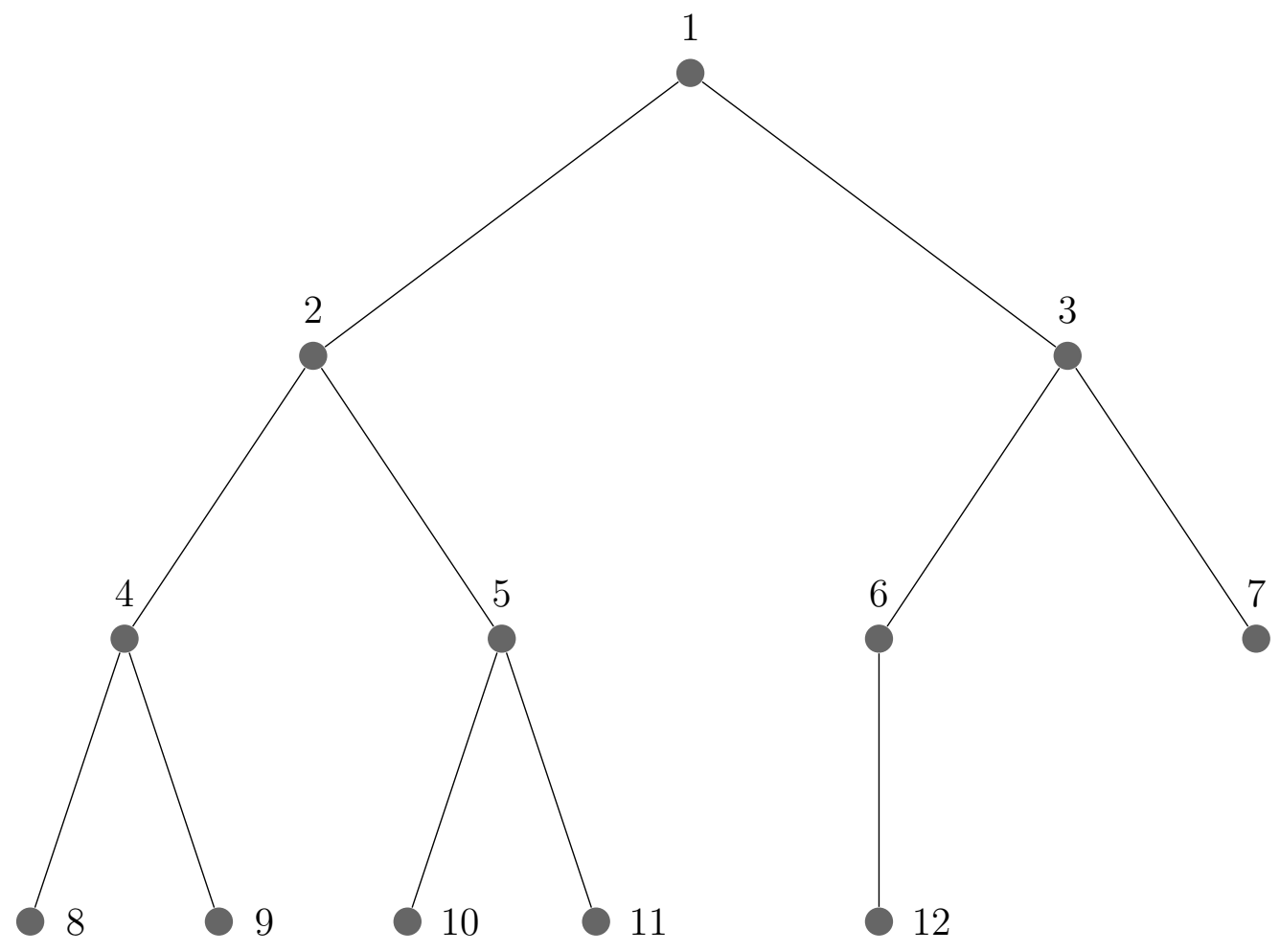

Figura 1.3: árvore dos vértices numerados de 1 a 12

Para esta árvore finita, temos $g(i)=3$ se $i \in\{2,3,4,5\}, g(i)=2$ se $i \in\{1,6\}$ e $g(i)=1$ se $i \in\{7,8,9,10,11,12\}$.

Agora, vamos definir o que é uma $n$-árvore e estudar alguns conceitos relacionados a este tipo de árvore.

Definição 1.5. Uma n-árvore ou árvore n-área completa é uma árvore com raiz onde cada vértice de qualquer nível está conectado a n vértices do próximo nível, exceto pelos vértices que estão no último nível da árvore.

Observação 1.5. A origem $\boldsymbol{O}$ é o nível 0 da árvore. O nível de cada vértice da árvore é igual a sua distância em relação a origem. 
Exemplo 1.5. A figura abaixo representa uma $n$-árvore de raiz $\omega$, com $n=2$.

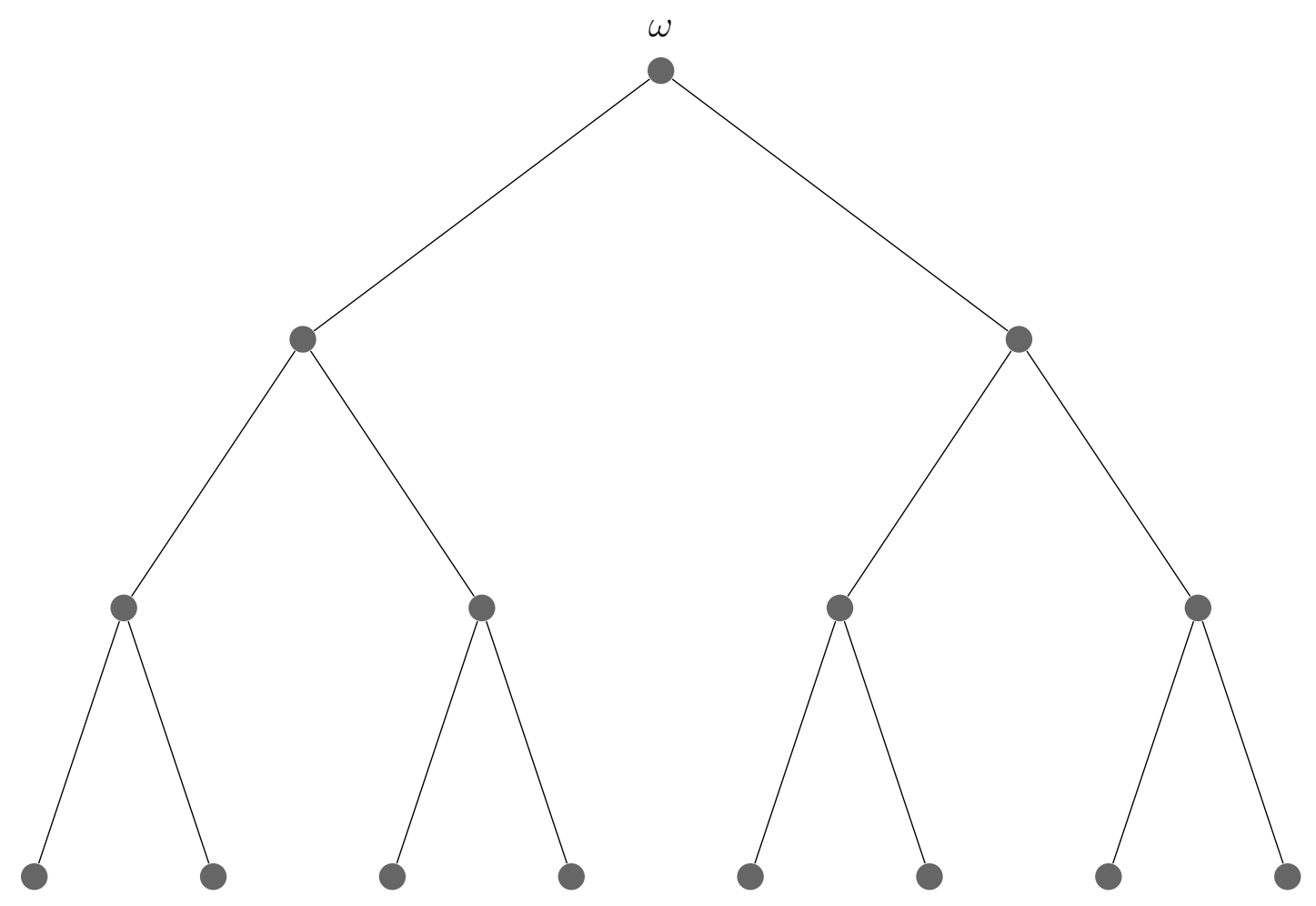

Figura 1.4: 2-árvore de raiz $\omega$

Definição 1.6. A altura de uma n-árvore é definida como a distância da raiz até o último nível da árvore. Denotamos essa altura por h. Daí, qualquer caminho da raiz ao último nível da árvore consiste de $h+1$ vértices.

Exemplo 1.6. Considere a Figura 1.4. Neste caso, temos a altura da 2-árvore igual a 3 e que cada caminho da raiz $\omega$ ao último nível da árvore consiste de exatamente 4 vértices.

Proposição 1.1. Seja $T=(\vartheta, \xi)$ uma árvore $n$-área. Então, o número de vértices do último nível de $T$ é igual a $n^{h}$.

Demonstração. A prova é por indução sobre $h$. Com efeito, para $h=1$ temos o último nível de $T$ com exatamente $n$ vértices. Agora, suponha válido para $h$. Devemos mostrar válido para $h+1$. De fato, cada vértice do nível $h$ está conectado a $n$ vértices do nível $h+1$. Como, por hipótese de indução, existem $n^{h}$ vértices no nível $h$ concluímos que existem $n \cdot n^{h}=n^{h+1}$ vértices no nível $h+1$. 
Observação 1.6. Cada vértice do último nível corresponde a um único caminho partindo da raiz. Logo, o número de caminhos partindo da raiz que conectam a algum dos vértices do último nível da $n$-árvore é igual a $n^{h}$.

Agora, vamos definir o que é uma árvore esfericamente simétrica, sua função de crescimento e um exemplo deste tipo de árvore.

Definição 1.7. Seja uma árvore infinita, localmente finita, $T=(\vartheta, \xi)$ com raiz $\boldsymbol{0}$. Dizemos que T é uma árvore esfericamente simétrica se qualquer par de vértices que tem a mesma distância da origem, têm o mesmo grau. Em outras palavras, T é uma árvore esfericamente simétrica se o grau de qualquer vértice $v$ de $\vartheta$ depende apenas da sua distância da raiz $\boldsymbol{O}$.

Observação 1.7. 1. Por definição, para cada $v$ de $\vartheta$, temos $|v|=d(\boldsymbol{O}, v)$;

2. Na árvore esfericamente simétrica, com raiz $\boldsymbol{O}$, vale que $g(v)=f(|v|)+1$, onde $f:=(f(i))_{i \geq 0}$ é uma sequência de inteiros positivos e $f$ é denominada função crescimento de $T$. 
Exemplo 1.7. Seja a árvore esfericamente simétrica $T$, abaixo.

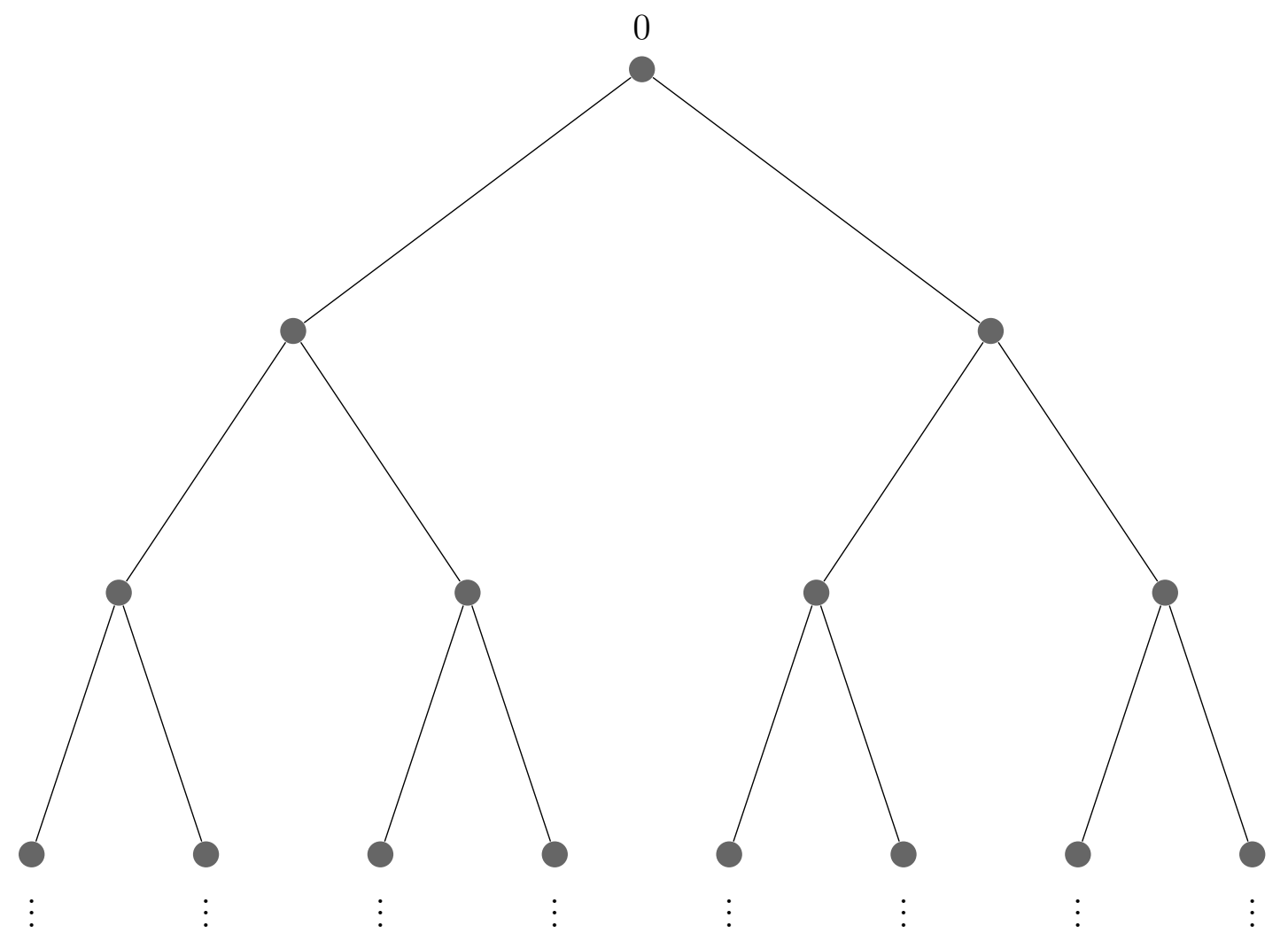

Figura 1.5: árvore esféricamente simétrica $T$

Para esta árvore, temos a função de crescimento dada por $f(i)=2, \forall i \geq 0$. Logo, $g(v)=f(|v|)+1=3, \forall v \in \vartheta \operatorname{com} v \neq \boldsymbol{O}$.

Agora, para cada $n>1$, seja $\partial T_{n}=\{v \in \vartheta ;|v|=n\}$.

Proposição 1.2. Seja T uma árvore esfericamente simétrica. Então, $\left|\partial T_{n}\right|=\prod_{i=0}^{n-1} f(i)$.

Demonstração. Indução sobre $n$. Para $n=1$ é válido, pois $\left|\partial T_{1}\right|=f(0)$. Suponha válido para $n$, ou seja, $\left|\partial T_{n}\right|=\prod_{i=0}^{n-1} f(i)$. Logo, $\left|\partial T_{n+1}\right|=\left|\partial T_{n}\right| \cdot f(n)=\left(\prod_{i=0}^{n-1} f(i)\right) \cdot f(n)=$ $\prod_{i=0}^{n} f(i)$. 
Definição 1.8. Uma árvore esfericamente simétrica infinita cuja função de crescimente é dada por $f(i)=i+1, \forall i \geq 0$, é dita árvore fatorial. Denotamos por $T$. a árvore fatorial. A figura abaixo, é uma representação da árvore $T$ !.

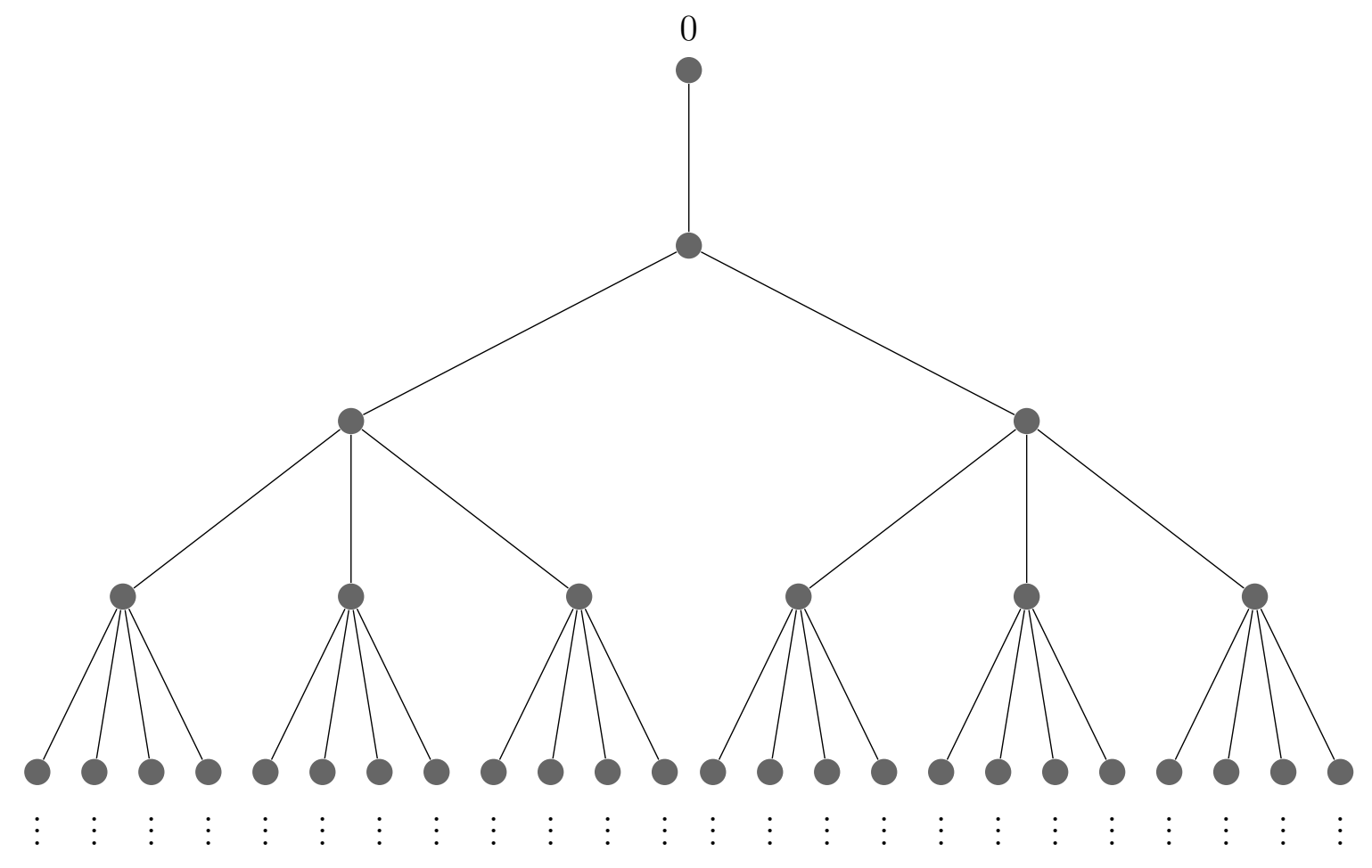

Figura 1.6: Uma representação da árvore $T_{!}$, até o nível $i=4$

Observação 1.8. Pela proposição 1.2 , segue que $\left|\partial T_{!, n}\right|=1.2 .3 \ldots n=n !$. 


\section{Capítulo 2}

\section{Modelo de percolação acessível em árvores $n$-árias}

Neste capítulo vamos estudar o modelo de percolação acessível na $n$-árvore. O modelo consiste em associar uma variável aleatória contínua $X_{v}$ para cada vértice $v$ da $n$ árvore. Consideremos que as variáveis aleatórias contínuas $X_{v}$ tem distribuição uniforme $(0,1)$. Suporemos, sem perda de generalidade, que para a raiz $\mathbf{0}$ atribuímos o valor 0. Vamos estudar sob quais condições existe pelo menos um caminho acessível na $n$-árvore, definindo assim a percolação acessível. Começaremos definindo o modelo e posteriormente estudando o número de caminhos acessíveis na $n$-árvore.

\section{$2.1 \quad$ O modelo}

Para este modelo, a cada vértice $v$ da árvore associamos uma variável aleatória $X_{v}$, tal que essa variável aleatória pertence a uma família de variáveis aleatórias contínuas $\left(X_{v}\right)_{v \in \vartheta}$ i.i.d. Chamamos modelo de percolação acessível o par formado pela árvore $T$ e a família de variáveis aleatórias contínuas $\left(X_{v}\right)_{v \in \vartheta}$. 
Definição 2.1. Um caminho de tamanho $n, v_{0}, v_{1}, v_{2}, \ldots . ., v_{n}$ em $T$ é acessível se

$$
X_{v_{0}}<X_{v_{1}}<X_{v_{2}}<X_{v_{3}}<\ldots<X_{v_{n}}
$$

Denotamos este evento por $v_{0} \overbrace{\rightarrow}^{c . a} v_{n}$

Exemplo 2.1. Seja a árvore 2-árvore T, abaixo.

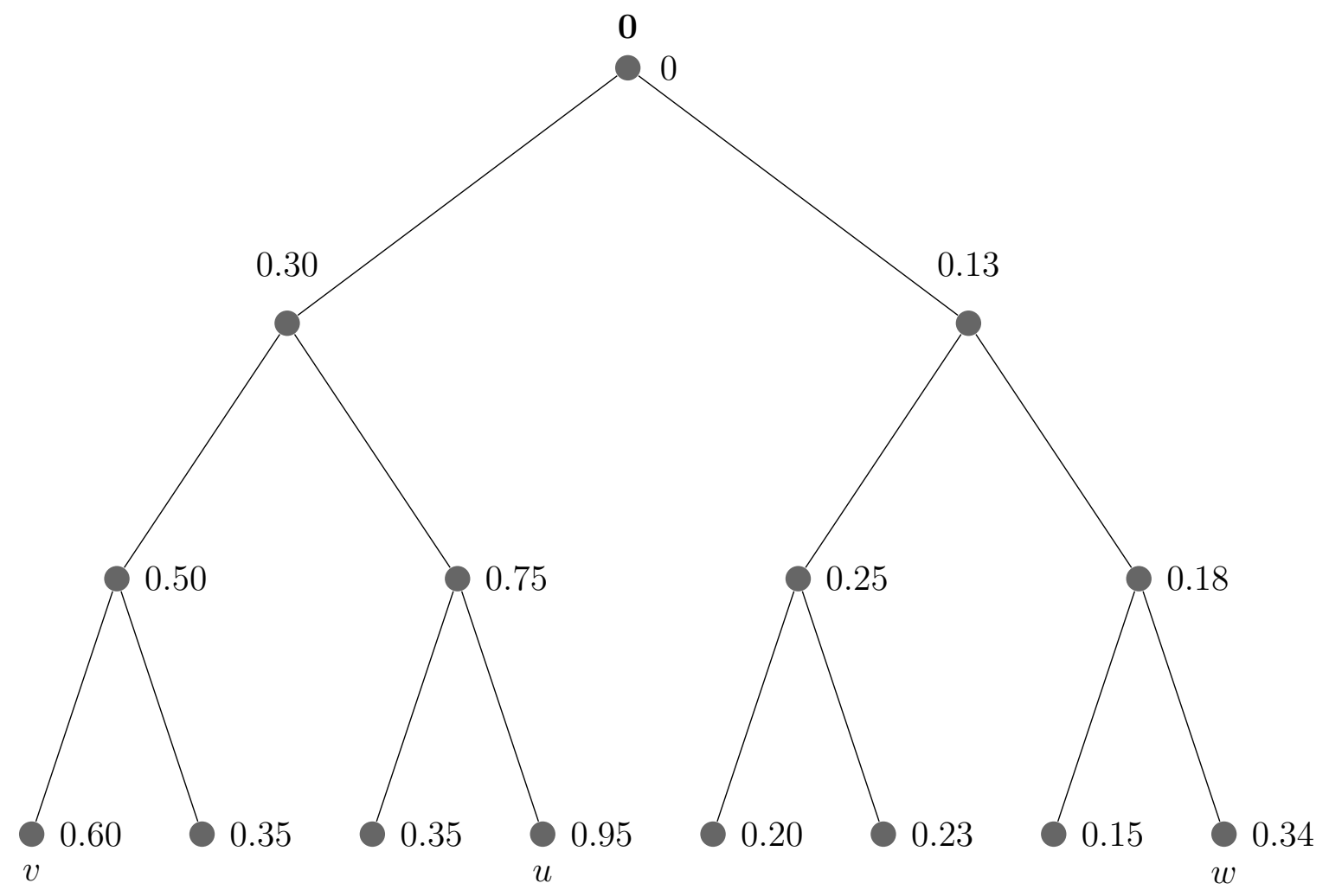

Figura 2.1: realizacão de um modelo de percolação acessível na 2-árvore $T$

A figura 2.1 é uma realização de um modelo de percolação acessível, tal que para cada $v \in \vartheta$ associamos $X_{v} \sim \mathbf{U}(0,1)$ de uma sequência i.i.d. Neste caso, temos que os caminhos $\boldsymbol{O} \rightarrow v, \boldsymbol{O} \rightarrow$ e e $\boldsymbol{O} \rightarrow w$ são caminhos acessíveis em $T$. 
O próximo resultado nos fornece a probabilidade exata de um caminho de tamanho $h$ ser acessível.

Proposição 2.1. Seja um caminho $v_{1}, v_{2}, \ldots ., v_{h}$, na árvore T. Então, $\mathbb{P}(v_{1} \overbrace{\rightarrow}^{\text {c.a }} v_{h})=\frac{1}{h !}$. Demonstração. Seja um caminho $v_{1}, v_{2}, \ldots, v_{h}$ na árvore $T$, tal que $X_{v_{i}}$ está associado a $v_{i}$ para todo $i \in\{1,2, \ldots, h\}$. Vamos facilitar a notação escrevendo $X_{v_{i}} \equiv X_{i}$, para todo $i \in\{1,2, \ldots, h\}$. Existem $h$ ! ordenações de $X_{1}, X_{2}, \ldots, X_{h}$, ou seja, ao longo do caminho podemos organizar os valores atribuídos as variáveis aleatórias, após uma dada realização, de $h$ ! modos. Por exemplo, para uma dada realização do modelo de percolação uma dessas ordens é $X_{1}<X_{2}<\ldots<X_{h}$. Outra ordem possível é $X_{2}<X_{3}<\ldots<X_{h}<X_{1}$. Por simétria, desde que $X_{1}, X_{2}, \ldots, X_{h}$ são i.i.d (independentes e identicamente distribuidas), todas as possíveis ordenações tem a mesma probabibilidade de ocorrer, ou seja, $\frac{1}{h !}$, então por exemplo, $\mathbb{P}\left(X_{2}<X_{3}<\ldots<X_{h}<X_{1}\right)=\frac{1}{h !}$. Portanto, $\mathbb{P}(v_{1} \overbrace{\rightarrow}^{\text {c.a }} v_{h})=\frac{1}{h !}$.

Observação 2.1. Considere um caminho de tamanho h que começa na origem $\left(v_{0}\right.$ até $\left.v_{h}\right)$, na n-árvore. Como a variável aleatória associada a origem assume o valor 0 , segue pela proposição 2.1 que $\mathbb{P}(v_{0} \overbrace{\rightarrow}^{c . a} v_{h})=\mathbb{P}(v_{1} \overbrace{\rightarrow}^{c . a} v_{h})=\frac{1}{h !}$.

\section{$2.2 \quad$ Número de caminhos acessíveis}

Denotamos por $N=\#$ de caminhos acessíveis, conectando a origem ao nível $h$, na $n$-árvore . Nesta seção, computaremos o primeiro e segundo momento da váriavel aleatória $N$.

\subsubsection{Cálculo do primeiro momento de $N(\mathbb{E}\{N\})$}

Esta secão tem por objetivo computar o primeiro momento da variável aleatória $N$. Para isto, veremos a proposição a seguir que nos fornecerá o valor da $\mathbb{E}\{N\}$.

Proposição 2.2. Seja $N$ dado acima. Então, o primeiro momento de $N$ é dado por

$$
\mathbb{E}\{N\}=\frac{n^{h}}{h !}
$$


Demonstração. Vamos definir uma variável aleatória indicadora $\mathbb{I}_{i}$ para cada caminho, conectando a origem ao nível $h$, da $n$-árvore com $i \in\left\{1,2,3, \ldots, n^{h}\right\}$. Seja a variável aleatória

$$
\mathbb{I}_{i}=\left\{\begin{array}{l}
1, \text { se o i-ésimo caminho for acessível } \\
0, \text { c.c }
\end{array}\right.
$$

Por linearidade da esperança, temos

$$
\mathbb{E}\{N\}=\mathbb{E}\left\{\sum_{i=1}^{n^{h}} \mathbb{I}_{i}\right\}=\sum_{i=1}^{n^{h}}\left(\mathbb{E}\left\{\mathbb{I}_{i}\right\}\right)=\sum_{i=1}^{n^{h}} \mathbb{P}\left(\mathbb{I}_{i}=1\right)=\sum_{i=1}^{n^{h}} \frac{1}{h !}=n^{h} \cdot \frac{1}{h !}=\frac{n^{h}}{h !}
$$

Exemplo 2.2. Considere a Figura 2.1. Para este caso, temos $\mathbb{E}\{N\}=\frac{n^{h}}{h !}=\frac{2^{3}}{3 !}=\frac{8}{6}=\frac{4}{3}$.

\subsubsection{Cálculo do segundo momento de $N$}

Está seção tem por objetivo encontrar um limitante superior para o segundo momento da variável aleatória $N$.

Proposição 2.3. Seja $\mathbb{E}\left\{N^{2}\right\}$ o segundo momento da variável aleatória $N$. Então, $\mathbb{E}\left\{N^{2}\right\} \leq \mathbb{E}\{N\}+\mathbb{E}\{N\}^{2}+\sum_{k=1}^{h-1}\left(\begin{array}{c}2 k \\ k\end{array}\right) \frac{n^{h+k}}{(h+k) !}$.

Demonstração. Vamos usar as variáveis $\mathbb{I}_{i}$ da proposição 2.2. Ou seja,

$$
\mathbb{I}_{i}=\left\{\begin{array}{l}
1, \text { se o i-ésimo caminho for acessível } \\
0, \text { c.c }
\end{array}\right.
$$

Note que, as variáveis aleatórias $\mathbb{I}_{i}, i \in\left\{1,2,3, \ldots, n^{h}\right\}$, são dependentes e identicamente distribuidas, tais que $\mathbb{E}\left\{\mathbb{I}_{i}\right\}=\mathbb{E}\left\{\mathbb{I}_{i}{ }^{2}\right\}=\mathbb{P}\left(\mathbb{I}_{i}=1\right)=\frac{1}{h !}$. Agora, considere que $N=\sum_{i=1}^{n^{h}} \mathbb{I}_{i}$. Logo, 


$$
\begin{aligned}
\mathbb{E}\left\{N^{2}\right\} & =\mathbb{E}\left\{\left(\sum_{i=1}^{n^{h}} \mathbb{I}_{i}\right)^{2}\right\} \\
& =\sum_{i=1}^{n^{h}} \mathbb{E}\left\{\mathbb{I}_{i}{ }^{2}\right\}+\sum_{i, j=1, i \neq j}^{n^{h}} \mathbb{E}\left\{\mathbb{I}_{i} \mathbb{I}_{j}\right\} \\
& =\sum_{i=1}^{n^{h}} \mathbb{E}\left\{\mathbb{I}_{i}\right\}+\sum_{i, j=1, i \neq j}^{n^{h}} \mathbb{E}\left\{\mathbb{I}_{i} \mathbb{I}_{j}\right\} \\
& =\mathbb{E}\{N\}+\sum_{i, j=1, i \neq j}^{n^{h}} \mathbb{E}\left\{\mathbb{I}_{i} \mathbb{I}_{j}\right\}
\end{aligned}
$$

Agora, vamos obter uma forma fechada para o correlacionador $\mathbb{E}\left\{\mathbb{I}_{i} \mathbb{I}_{j}\right\}$. Primeiramente, notemos que este correlacionador depende apenas do número de vértices que o $i$-ésimo e o $j$-ésimo caminho tem em comum, pois se o $i$-ésimo e o $j$-ésimo caminhos não possuem vértices em comum, temos $\mathbb{E}\left\{\mathbb{I}_{i} \mathbb{I}_{j}\right\}=\mathbb{E}\left\{\mathbb{I}_{i}\right\} \mathbb{E}\left\{\mathbb{I}_{j}\right\}$, por outro lado se o $i$-ésimo e o $j$-ésimo caminhos compartilham vértices, temos $\mathbb{E}\left\{\mathbb{I}_{i} \mathbb{I}_{j}\right\}=\mathbb{E}\left\{\mathbb{E}\left\{\mathbb{I}_{i} \mathbb{I}_{j} / \mathbb{I}_{J}\right\}\right\}$. Note também que para quaisquer dois caminhos $i$ e $j($ com $i \neq j$ ) que compartilham $h-k+1$ vértices, $\mathbb{E}\left\{\mathbb{I}_{i} \mathbb{I}_{j}\right\}$ é dado pela probabilidade $\pi_{k}$ de ambos os caminhos serem acessíveis, ou seja, $\mathbb{E}\left\{\mathbb{I}_{i} \mathbb{I}_{j}\right\}=\mathbb{P}\left(\mathbb{I}_{i} \mathbb{I}_{j}=1\right)=\mathbb{P}\left(\mathbb{I}_{i}=1 ; \mathbb{I}_{j}=1\right)=\pi_{k}$. Seja $x$ o valor atribuido a $X_{v}$, onde $v$ é o vértice de divergência dos dois caminhos. Notemos que, estes dois caminhos podem ser decompostos em três caminhos menores e independentes, dois de tamanhos $k$ e um de tamanho $h-k$ conectados ao vértice $v$ (veja figura 2.2).

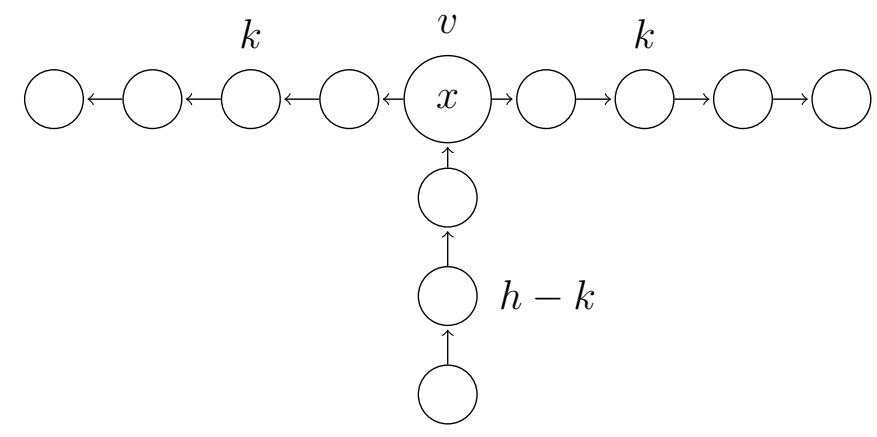

Figura 2.2: A correlação entre dois caminhos depende apenas do número $h-k+1$ de vértices que ambos os caminhos têm em comum.

Considerando $v$ como raiz, todos os vértices que estão mais próximos de $v$ devem ter um valor atribuido menor que $x$ e todos os vértices mais próximos aos últimos níveis da árvore devem ter um valor maior que $x$. Além disso, todos os valores devem estar 
em ordem crescente nesses caminhos menores. Daí, usando o fato de que as variáveis aleatórias tem distribuição uniforme $(0,1)$, concluímos que

$$
\begin{aligned}
\pi_{k} & =\mathbb{P}\left(\mathbb{I}_{i}=1 ; \mathbb{I}_{j}=1 / X_{v}=x\right) \\
& =\int_{0}^{1} \mathbb{P}\left(\mathbb{I}_{i}=1 ; \mathbb{I}_{j}=1 / X_{v}=x\right) f(x) d x \\
& =\int_{0}^{1} \mathbb{P}\left(X_{1}<X_{2}<\ldots<X_{h-k-1}<x\right) \mathbb{P}\left(x<X_{h-k+1}<\ldots<X_{h}\right)^{2} d x \\
& =\int_{0}^{1} \frac{[F(x)]^{h-k-1}}{(h-k-1) !}\left[\frac{(1-F(x))^{k}}{k !}\right]^{2} d x \\
& =\int_{0}^{1} \frac{x^{h-k-1}}{(h-k-1) !}\left[\frac{(1-x)^{k}}{k !}\right]^{2} d x \\
& =\frac{1}{(h-k-1) !(k !)^{2}} \int_{0}^{1} x^{h-k-1}(1-x)^{2 k} d x \\
& =\frac{B(h-k, 2 k+1)}{(h-k-1) !(k !)^{2}} \\
& =\frac{(h-k-1) !(2 k) !}{(h+k) !}\left(\frac{1}{(h-k-1) !(k !)^{2}}\right) \\
& =\left(\begin{array}{c}
2 k \\
k
\end{array}\right) \frac{1}{(h+k) !}
\end{aligned}
$$

onde $B(x, y)=\int_{0}^{1} t^{x-1}(1-t)^{y-1}=\frac{(x-1) !(y-1) !}{(x+y-1) !}$ é uma função beta de Euler.

Para avaliar a soma sobre a correlação entre dois caminhos $i$ e $j$ em $\pi_{k}$ também é necessário o número $m_{k}$ de pares de caminhos que possuem $h-k+1$ vértices comuns. Este número pode ser avaliado com uma simples combinatória, considerando que: Para o primeiro caminho entre os três na Figura 2.2, digamos o de tamanho $k$, qualquer vértice pode ser escolhido, isto é, existem $n^{h}$ possibilidades ( que é o número de caminhos da árvore). O segundo caminho compartilha $h-k+1$ vértices, então existem $n-1$ vértices, que dista 1 de $x$ em potencial para escolher (se o $n$-ésimo vértice, também pertence ao primeiro caminho) e finalmente, pode-se escolher qualquer vértice subsequente até que se alcance o último nível de outro caminho e isso equivale a $n^{k-1}$ possibilidades. Logo, existem ao todo $m_{k}=(n-1) n^{h} n^{k-1}=(n-1) n^{h+k-1}$ pares diferentes. Consequentemente, 


$$
\begin{aligned}
\mathbb{E}\left\{N^{2}\right\} & =\mathbb{E}\{N\}+\sum_{i, j=1, i \neq j}^{n^{h}} \mathbb{E}\left\{\mathbb{I}_{i} \mathbb{I}_{j}\right\} \\
& =\mathbb{E}\{N\}+\sum_{k=1}^{h} \pi_{k} m_{k} \\
& =\mathbb{E}\{N\}+\frac{n-1}{n} \sum_{k=1}^{h}\left(\begin{array}{c}
2 k \\
k
\end{array}\right) \frac{n^{h+k}}{(h+k) !} \\
& \leq \mathbb{E}\{N\}+\left(\begin{array}{c}
2 h \\
h
\end{array}\right) \frac{n^{2 h}}{(2 h) !}+\sum_{k=1}^{h-1}\left(\begin{array}{c}
2 k \\
k
\end{array}\right) \frac{n^{h+k}}{(h+k) !}, \text { pois } \frac{n-1}{n} \leq 1 \\
& =\mathbb{E}\{N\}+\mathbb{E}\{N\}^{2}+\sum_{k=1}^{h-1}\left(\begin{array}{c}
2 k \\
k
\end{array}\right) \frac{n^{h+k}}{(h+k) !} .
\end{aligned}
$$

Portanto,

$$
\mathbb{E}\left\{N^{2}\right\} \leq \mathbb{E}\{N\}+\mathbb{E}\{N\}^{2}+\sum_{k=1}^{h-1}\left(\begin{array}{c}
2 k \\
k
\end{array}\right) \frac{n^{h+k}}{(h+k) !}
$$

\subsection{Probabilidade de existir caminhos acessíveis na $n$-árvore}

Nesta seção, vamos usar o primeiro e o segundo momento da variável aleatória $N$ para obter informações sobre a existência de caminhos acessíveis na $n$-árvore. Vejamos primeiramente a desigualdade de Cauchy-Schwarz e depois um lema, que relaciona o primeiro e o segundo momento de uma variável aleatória $X$ que assume valores inteiros $\{0,1,2, \ldots\}$.

Lema 2.1 (desigualdade de Cauchy-Schwarz). Se $X$ e $Y$ são variáveis aleatórias com segundo momento finito, então $|\mathbb{E}\{X Y\}| \leq \sqrt{\mathbb{E}\left\{X^{2}\right\} \mathbb{E}\left\{Y^{2}\right\}}$. 
Lema 2.2. Seja $X$ uma variável aleatória que assume apenas valores inteiros $\{0,1,2, \ldots .$. com segundo momento finito. Então vale a inequação

$$
\mathbb{E}\{X\} \geq \mathbb{P}(X \geq 1) \geq \frac{\mathbb{E}\{X\}^{2}}{\mathbb{E}\left\{X^{2}\right\}}
$$

Demonstração. Por definição da esperança, temos

$$
\mathbb{E}\{X\}=\sum_{i=0}^{\infty} i \mathbb{P}(X=i) \geq\left[\sum_{i=1}^{\infty} \mathbb{P}(X=i)\right]=\mathbb{P}(X \geq 1)
$$

Agora, vamos mostrar que $\mathbb{P}(X \geq 1) \geq \frac{\mathbb{E}\{X\}^{2}}{\mathbb{E}\left\{X^{2}\right\}}$. Com efeito, usando a desigualdade de Cauchy-Schwarz, temos $\mathbb{E}\{X\}=\left|\mathbb{E}\left\{X I_{\{X \geq 1\}}\right\}\right| \leq \sqrt{\mathbb{E}\left\{X^{2}\right\} \mathbb{E}\left\{I_{\{X \geq 1\}}\right\}}$. Logo, $\mathbb{E}\left\{X^{2}\right\} \mathbb{P}(X \geq 1) \geq \mathbb{E}\{X\}^{2}$. Consequentemente,

$$
\mathbb{P}(X \geq 1) \geq \frac{\mathbb{E}\{X\}^{2}}{\mathbb{E}\left\{X^{2}\right\}}
$$

Portanto, a combinação de (2.4) e (2.5) nos fornece o resultado desejado, ou seja,

$$
\mathbb{E}\{X\} \geq \mathbb{P}(X \geq 1) \geq \frac{\mathbb{E}\{X\}^{2}}{\mathbb{E}\left\{X^{2}\right\}}
$$

Como consequência imediata do Lema 2.2, concluímos que combinando as desigualdades (2.2) e (2.3), obtemos

$$
\mathbb{E}\{N\} \geq \mathbb{P}(N \geq 1) \geq \frac{\mathbb{E}\{N\}^{2}}{\mathbb{E}\left\{N^{2}\right\}} \geq \frac{\mathbb{E}\{N\}^{2}}{\mathbb{E}\{N\}+\mathbb{E}\{N\}^{2}+S(h)},
$$

onde

$$
S(h)=\sum_{k=1}^{h-1}\left(\begin{array}{c}
2 k \\
k
\end{array}\right) \frac{n^{h+k}}{(h+k) !}
$$


Vamos tratar o número de ramificações $n$ como uma função de $h$. Já que vamos distinguir os três casos de crescimento linear, crescimento mais rápido que linear e crescimento mais lento que linear, nós escrevemos

$$
n=n(h)=h \alpha(h)
$$

Os três casos acima correspondem a:

1. $\alpha(h)=\alpha=$ constante (crescimento constante da árvore);

2. $\alpha(h) \rightarrow \infty$ (expansão muito rápido das laterais); e

3. $\alpha(h) \rightarrow 0$ (compressão das laterais).

Além disso, suponhamos que $n(h+1) \geq n(h)$. Como $\mathbb{E}\{N\}=\frac{n^{h}}{h !}$, vamos usar a fórmula de Stirling

$$
n ! \sim \sqrt{2 \pi n}\left(\frac{n}{e}\right)^{n}
$$

para obter um valor médio para $N$. Logo,

$$
\mathbb{E}\{N\} \approx \frac{n(h)^{h}}{\sqrt{2 \pi h} h^{h} e^{-h}}=\frac{[e \alpha(h)]^{h}}{\sqrt{2 \pi h}}
$$

Pela relação (2.7), concluímos o seguinte:

1. $\mathbb{E}\{N\} \approx 0$ se $\alpha(h) \rightarrow 0$ ou $\alpha(h) \equiv \alpha \leq e^{-1}$

2. $\mathbb{E}\{N\} \rightarrow \infty$ se $\alpha(h) \rightarrow \infty$ ou $\alpha(h) \equiv \alpha>e^{-1}$.

Primeiro, vamos considerar o caso $\alpha(h) \rightarrow 0$ para mostrarmos que $S(h)$ cresce mais lentamente que $\mathbb{E}\{N\}$. Para isto, vejamos a proposição a seguir. 
Proposição 2.4. Sejam $S(h)=\sum_{k=1}^{h-1}\left(\begin{array}{c}2 k \\ k\end{array}\right) \frac{n^{h+k}}{(h+k) !}$ e $\mathbb{E}\{N\}=\frac{n^{h}}{h !}$. Então, $S(h) \cdot \mathbb{E}\{N\}^{-1} \leq 0$ para $h$ suficientemente grande. Consequentemente, $\mathbb{P}(N \geq 1)$ é assintóticamente equivalente a $\mathbb{E}\{N\}$.

Demonstração. Aplicando a fórmula de Stirling em $S(h)$, obtemos $\left(\begin{array}{c}2 k \\ k\end{array}\right) \leq \frac{4^{k}}{\sqrt{\pi k}}, \operatorname{logo}$

$$
\begin{aligned}
S(h) & =\sum_{k=1}^{h-1}\left(\begin{array}{c}
2 k \\
k
\end{array}\right) \frac{n^{h+k}}{(h+k) !} \\
& =\mathbb{E}\{N\} \sum_{k=1}^{h-1}\left(\begin{array}{c}
2 k \\
k
\end{array}\right) \frac{n^{k}}{(h+k) \cdot(h+k-1) \ldots(h+1)} \text {, aplicando Stirling } \\
& \leq \mathbb{E}\{N\} \sum_{k=1}^{h-1} \frac{(4 n)^{k}}{\sqrt{\pi k}(h+k) \cdot(h+k-1) \ldots(h+1)} \\
& =\mathbb{E}\{N\}(x)\left(\frac{1-x^{h-1}}{1-x}\right)
\end{aligned}
$$

com $x=\frac{4 n}{h+1}$. Daí, $S(h) . \mathbb{E}\{N\}^{-1} \leq(x)\left(\frac{1-x^{h-1}}{1-x}\right)$. Logo, para $h$ suficientemente grande, quando $x \rightarrow 0$, concluímos que $S(h) . \mathbb{E}\{N\}^{-1} \leq 0$. Ou seja, $S(h) \rightarrow 0$. Consequentemente, pela desigualdade (2.6), temos $\lim _{h \rightarrow \infty} \frac{\mathbb{P}(N \geq 1)}{\mathbb{E}\{N\}}=1$, i.é, $\mathbb{P}(N \geq 1)$ é assintóticamente equivalente a $\mathbb{E}\{N\}$.

Para o caso $\alpha(h) \rightarrow \infty$ é um pouco mais complicado. Semelhantemente ao caso anterior, mostraremos que $S(h)$ cresce mais lentamente do que $\mathbb{E}\{N\}^{2}$, o que vai implicar em $\mathbb{P}(N \geq 1) \rightarrow 1$ quando $h \rightarrow \infty$. Para isto, vamos demonstrar a proposição seguir. 
Proposição 2.5. Seja a função

$$
\xi(h)=S(h) \mathbb{E}\{N\}_{h}^{-2}
$$

Então,

$$
\xi(h+1) \leq \frac{1+\xi(h)}{\alpha(h)}
$$

Demonstração. Consideremos que $n(h+1) \geq n(h)$ e $n(h)=h \alpha(h)$. Portanto,

$$
\begin{aligned}
& \xi(h+1)=S(h+1) \mathbb{E}\{N\}_{h+1}^{-2} \\
& =\frac{[(h+1) !]^{2}}{[n(h+1)]^{2(h+1)}} \sum_{k=1}^{h}\left(\begin{array}{c}
2 k \\
k
\end{array}\right) \frac{[n(h+1)]^{h+k+1}}{(h+k+1) !} \\
& =\frac{[(h+1) !]^{2}}{[n(h+1)]^{h+1}} \sum_{k=1}^{h}\left(\begin{array}{c}
2 k \\
k
\end{array}\right) \frac{[n(h+1)]^{k}}{(h+k+1) !} \\
& \leq \frac{[(h+1) !]^{2}}{[n(h)]^{h+1}} \sum_{k=1}^{h}\left(\begin{array}{c}
2 k \\
k
\end{array}\right) \frac{[n(h)]^{k}}{(h+k+1) !} \text {, pois } n(h+1) \geq n(h) \\
& =\frac{[(h+1) !]^{2}}{[n(h)]^{h+1}}\left[\frac{[n(h)]^{h}}{(2 h+1)(2 h) !} \frac{(2 h) !}{(h !)^{2}}+\frac{1}{[n(h)]^{h}(h+2)} \sum_{k=1}^{h-1}\left(\begin{array}{c}
2 k \\
k
\end{array}\right) \frac{[n(h)]^{h+k}}{(h+k) !}\right] \\
& =\frac{(h+1)^{2}}{(2 h+1) n(h)}+\frac{(h+1)^{2}(h !)^{2}}{[n(h)]^{2 h+1}(h+2)} S(h) \\
& =\frac{1}{n(h)}\left[\frac{(h+1)^{2}}{(2 h+1)}+\frac{(h+1)^{2}(h !)^{2}}{[n(h)]^{2 h}(h+2)} S(h)\right] \\
& =\frac{1}{\alpha(h)}\left[\frac{(h+1)^{2}}{h(2 h+1)}+\frac{(h+1)^{2}}{h(h+2)}\left(\frac{n(h)^{h}}{h !}\right)^{-2} S(h)\right] \text {, pois } n(h)=h \alpha(h) \\
& =\frac{1}{\alpha(h)}\left[\frac{(h+1)^{2}}{h(2 h+1)}+\frac{(h+1)^{2}}{h(h+2)} S(h) \mathbb{E}\{N\}_{h}{ }^{-2}\right] \\
& =\frac{1}{\alpha(h)}\left[\frac{(h+1)^{2}}{h(2 h+1)}+\frac{(h+1)^{2}}{h(h+2)} \xi(h)\right] \\
& \leq \frac{1}{\alpha(h)}(1+\xi(h)) \\
& =\frac{1+\xi(h)}{\alpha(h)}
\end{aligned}
$$


Agora, desde que $\alpha(h) \rightarrow \infty$, podemos tomar uma constante fixa $C>1$ e encontrar $h_{0}$ tal que $\alpha(h)>C, \forall h>h_{0}$. Daí, teremos

$$
\xi(h+1) \leq \frac{1+\xi(h)}{\alpha(h)}<\frac{\xi(h)}{C}+\frac{1}{C}, \forall h>h_{0} .
$$

Agora, mostraremos por indução que

$$
\xi(h) \leq \frac{\xi\left(h_{0}\right)}{C^{h-h_{0}}}+\sum_{k=1}^{h-h_{0}} \frac{1}{C^{k}}
$$

Para $k=1, \operatorname{temos} \frac{\xi\left(h_{0}\right)}{C^{h-h_{0}}}+\sum_{k=1}^{h-h_{0}} \frac{1}{C^{k}}=\frac{\xi\left(h_{0}\right)}{C^{1}}+\sum_{k=1}^{1} \frac{1}{C^{1}}=\frac{\xi\left(h_{0}\right)}{C}+\frac{1}{C}>\xi(h)$. Suponha válido para $k$, ou seja, $\xi(h) \leq \frac{\xi\left(h_{0}\right)}{C^{h-h_{0}}}+\sum_{k=1}^{h-h_{0}} \frac{1}{C^{k}}$ é verdadeiro. Logo, $\frac{\xi\left(h_{0}\right)}{C^{h-h_{0}}}+\sum_{k=0}^{h-h_{0}} \frac{1}{C^{k+1}} \geq \frac{\xi\left(h_{0}\right)}{C^{h-h_{0}}}+\sum_{k=1}^{h-h_{0}} \frac{1}{C^{k}} \geq \xi(h)$. Portanto, a desigualdade (2.10) é verdadeira.

Notemos que quando $h \rightarrow \infty$, temos

$$
\sum_{k=1}^{h-h_{0}} \frac{1}{C^{k}} \rightarrow \frac{1}{C-1}
$$

Portanto, para $C$ arbitrariarmente grande concluímos que $\xi(h) \rightarrow 0$. Consequentemente, concluímos pela desigualdade $(2.7)$ que $\mathbb{P}(N \geq 1) \rightarrow 1$ quando $h \rightarrow \infty$.

Teorema 2.2. Suponha que $n=n(h)=h \alpha(h)$. Então,

$$
\lim _{h \rightarrow \infty} \mathbb{P}(N \geq 1)=\left\{\begin{array}{l}
0, \text { se } \alpha(h) \rightarrow 0 \\
1, \text { se } \alpha(h) \rightarrow \infty
\end{array}\right.
$$

Demonstração. A demonstração consiste na combinando das proposições 2.4 e 2.5. Portanto, o resultado segue.

Finalmente, vamos considerar o caso $\alpha(h)=\alpha=$ constante. Usando a forma recursiva dada por $(2.10)$, provaremos que $\xi(h) \leq(\alpha-1)^{-1}$ com $\alpha>1$. Para isto, veja a proposição a seguir. 
Proposição 2.6. Seja $\xi(h+1) \leq \frac{1+\xi(h)}{\alpha(h)}$. Então, $\xi(h) \leq(\alpha-1)^{-1} \operatorname{com} \alpha>1$.

Demonstração. Indução sobre $h$. Suponha válido para $h$, ou seja, $\xi(h) \leq(\alpha-1)^{-1}$. Devemos mostrar que $\xi(h+1) \leq(\alpha-1)^{-1}$. Com efeito,

$$
\xi(h+1) \leq \frac{1+\xi(h)}{\alpha(h)} \leq \frac{1+(\alpha-1)^{-1}}{\alpha}=\frac{1}{\alpha-1}=(\alpha-1)^{-1}
$$

Observação 2.2. Notemos que $\lim _{h \rightarrow \infty} \xi(h) \leq \lim _{h \rightarrow \infty}(\alpha-1)^{-1}=(\alpha-1)^{-1}$. Ou seja, $\xi(h)$ tem limite finito.

Agora, demonstraremos o resultado principal para o caso $\alpha(h)=\alpha=$ constante.

Teorema 2.3 (Nowak e Krug.(2013)). Se $n=\alpha h$, com $\alpha>0$ uma constante, então

$$
\lim _{h \rightarrow \infty} \mathbb{P}(N \geq 1)\left\{\begin{array}{l}
=0, \text { se } \alpha \leq e^{-1} \\
\geq 1-\alpha^{-1}, \text { se } \alpha>1
\end{array}\right.
$$

Demonstração. Primeiramente, vamos supor que $\alpha \leq e^{-1}$. Pela desigualdade (2.6) e a relação (2.7) concluímos que

$$
0 \geq \lim _{h \rightarrow \infty} \mathbb{P}(N \geq 1) \geq 0
$$

Logo, $\lim _{h \rightarrow \infty} \mathbb{P}(N \geq 1)=0$. Agora, suponha que $\alpha>1$. Pela desigualdade (2.6), obtemos

$$
\begin{aligned}
\lim _{h \rightarrow \infty} \frac{1}{\mathbb{P}(N \geq 1)} & \leq \lim _{h \rightarrow \infty} \frac{\mathbb{E}\{N\}+\mathbb{E}\{N\}^{2}+S(h)}{\mathbb{E}\{N\}^{2}} \\
& =\lim _{h \rightarrow \infty}\left(\frac{1}{\mathbb{E}\{N\}}+1+\frac{S(h)}{\mathbb{E}\{N\}^{2}}\right) \\
& =1+\lim _{h \rightarrow \infty} \xi(h) .
\end{aligned}
$$

Como $\lim _{h \rightarrow \infty} \xi(h) \leq(\alpha-1)^{-1}$, temos $\lim _{h \rightarrow \infty} \frac{1}{\mathbb{P}(N \geq 1)} \leq 1+(\alpha-1)^{-1}$. Consequentemente, $\lim _{h \rightarrow \infty} \mathbb{P}(N \geq 1) \geq \frac{1}{1+(\alpha-1)^{-1}}=\frac{\alpha-1}{\alpha}=1-\alpha^{-1}$. 


\section{Capítulo 3}

\section{Discussão}

Neste capítulo vamos discutir sobre outros resultados recentes do modelo de percolação. Vamos centralizar a discussão na prova complementar do teorema de Nowak e Krug, e posteriormente sobre o trabalho recente do modelo de percolação na árvore esfericamente simétrica infinita.

\subsection{Discussão}

No final do Capítulo 2 enunciamos e provamos o Teorema 2.3. Existe um resultado importante que completa este teorema. Notemos que, no Teorema 2.3 é razoável supor que o limite é uma sequência monótona em $\alpha$. Este fato pode ser verificado da seguinte forma: sejam duas árvores $n$-árias de altura $h$, digamos $T_{1}$ e $T_{2}$, associadas ao valores $\alpha_{1}$ e $\alpha_{2}$, respectivamente, com $\alpha_{1}<\alpha_{2}$. Suponhamos que $T_{1}$ é subárvore de $T_{2}$. Logo, todo caminho acessível em $T_{1}$ também o é em $T_{2}$. Daí, concluímos que $\mathbb{P}\left(N_{1} \geq 1\right) \leq$ $\mathbb{P}\left(N_{2} \geq 1\right)$. Consequentemente, $\lim _{h \rightarrow \infty} \mathbb{P}\left(N_{1} \geq 1\right) \leq \lim _{h \rightarrow \infty} \mathbb{P}\left(N_{2} \geq 1\right)$. Portanto, podemos supor o limite como uma sequência monótona em $\alpha$. Concluímos, devido a monoticidade da sequência formado por esse limite, que deve existir um valor crítico $\alpha_{c} \in\left[e^{-1}, 1\right]$, tal que $\mathbb{P}(N \geq 1) \rightarrow 0$ para $\alpha<\alpha_{c}$ e $\lim _{h \rightarrow \infty} \mathbb{P}(N \geq 1)>0$ para $\alpha>\alpha_{c}$. Este resultado pode ser encontrado em [2], no teorema de Roberts e Zhao, que diz: Se $n=\alpha h$, então $\alpha_{c}=e^{-1}$, i.e.,

$$
\lim _{h \rightarrow \infty} \mathbb{P}(N \geq 1)=\left\{\begin{array}{l}
0, \text { se } \alpha \leq e^{-1}, \\
1, \text { se } \alpha>e^{-1}
\end{array}\right.
$$


Agora, abordaremos alguns resultados mais recentes sobre percolação acessível obtidos em [3]. De acordo com o capítulo 1 , uma árvore $T=(\vartheta, \xi)$ é dita esfericamente simétrica se qualquer par de vértices na mesma distância da origem, têm o mesmo grau. O modelo consiste em associar uma variável aleatória contínua $X_{v}$ para cada vértice $v$ da árvore esfericamente simétrica infinita. Para o modelo em apreço, tomamos as $X_{v}$ como tendo distribuição uniforme $(0,1)(\mathbb{U}(0,1))$. Agora, para cada $n>1$, seja $\partial T_{n}=\{v \in \vartheta ;|v|=n\}$ e considere $\Lambda_{n}:=\Lambda_{n}(T)=\bigcup_{v \in \partial T_{n}}\{0 \overbrace{\rightarrow}^{\text {c.a }} v\}$. Dizemos que $N_{n}$ é o número de caminhos acessíveis conectando a origem ao $n$-ésimo nível da árvore $T$. Ou seja, $N_{n}=\left|\{v \in \partial T_{n} ; 0 \overbrace{\rightarrow}^{\text {c.a }} v\}\right|$. A definição a seguir, caracteriza quando há percolação acessível na árvore esfericamente simétrica.

Definição 3.1. Dizemos que há percolação acessível na árvore $T=(\vartheta, \xi)$ se o evento $\bigcap_{n=1}^{\infty} \Lambda_{n}$ ocorre com probabilidade positiva.

Observação 3.1. Note que $\left(\Lambda_{n}\right)_{n \in \mathbb{N}}$ é uma sequência decrescente de eventos $\left(\Lambda_{n+1} \subset \Lambda_{n}, \forall n, \Lambda_{n} \searrow \bigcap_{n=1}^{\infty} \Lambda_{n}\right)$, então o nosso interesse é estudar $\theta(T):=\mathbb{P}\left(\bigcap_{n=1}^{\infty} \Lambda_{n}\right)=\lim _{n \rightarrow \infty} \mathbb{P}\left(\Lambda_{n}\right)$.

Definição 3.2. Seja $T_{1}=\left(\vartheta_{1}, \xi_{1}\right)$ e $T_{2}=\left(\vartheta_{2}, \xi_{2}\right)$ duas árvores com comum origem $\boldsymbol{0}$. Nós dizemos que $T_{1}$ é sub-árvore de $T_{2}$, denotamos $T_{1} \prec T_{2}$, se $\vartheta_{1} \subset \vartheta_{1}$ e $\xi_{1} \subset \xi_{2}$. 
Lema 3.1. Se $T_{1} \prec T_{2}$, então $\theta\left(T_{1}\right) \leq \theta\left(T_{2}\right)$.

Demonstração. Basta mostrar que todo caminho acessível conectando $\mathbf{0}$ a $\partial T_{1, n}$ também é caminho acessível conectando 0 a $\partial T_{2, n}$. Com efeito, seja um caminho acessível $\mathbf{0} \overbrace{\rightarrow}^{\text {c.a }} v$ para algum $v \in \partial T_{1, n}$. Como, por hipótese, $T_{1} \prec T_{2}$, então $0 \stackrel{\text { c.a }}{\rightarrow} v$ é caminho acessível em $T_{2}$. Veja as árvores $T_{1}$ e $T_{2}$, respectivamente:

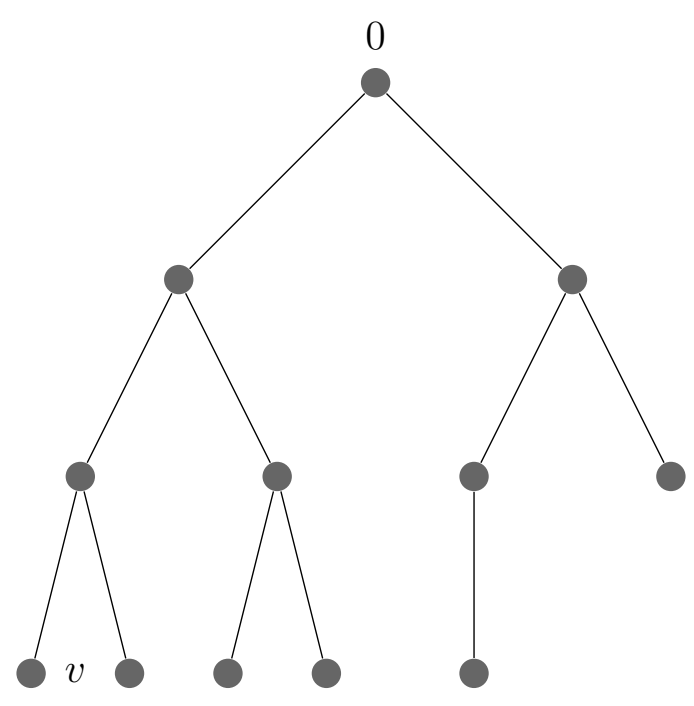

Figura 3.1: árvore $T_{1}$

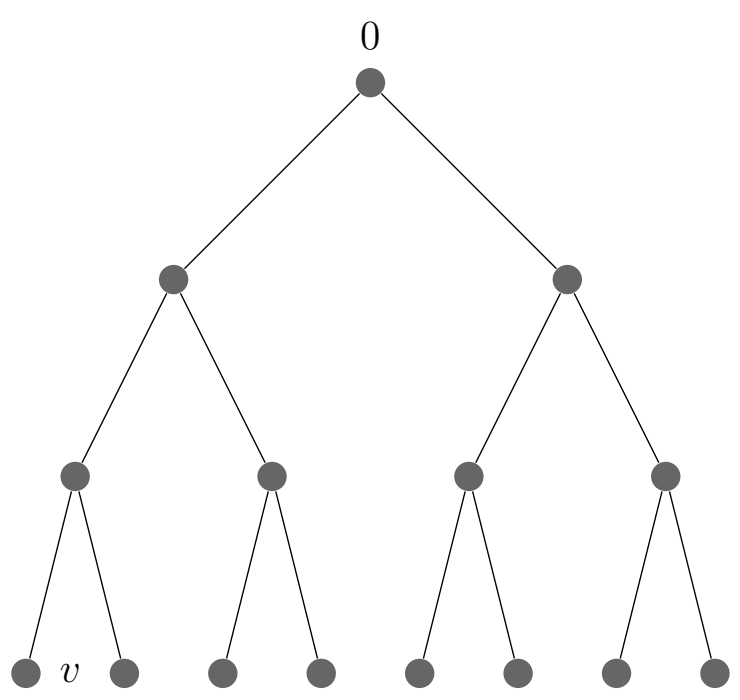

Figura 3.2: árvore $T_{2}$

Notemos que as Figuras 3.1 e 3.2 são duas árvores, tais que $T_{1}$ é sub-árvore de $T_{2}$. Portanto, como $\Lambda_{n}(T)=\bigcup_{v \in \partial T_{n}}\{0 \overbrace{\rightarrow}^{\text {c.a }} v\}$, temos $\Lambda_{n}\left(T_{1}\right) \subset \Lambda_{n}\left(T_{2}\right)$. Portanto, $P\left(\Lambda_{n}\left(T_{1}\right)\right) \leq P\left(\Lambda_{n}\left(T_{2}\right)\right)$ implicando que $\lim _{n \rightarrow \infty} P\left(\Lambda_{n}\left(T_{1}\right)\right) \leq \lim _{n \rightarrow \infty} P\left(\Lambda_{n}\left(T_{2}\right)\right)$ implicando que $\theta\left(T_{1}\right) \leq \theta\left(T_{2}\right)$.

Observação 3.2. $\theta(T)$ é uma função não-decrescente em $T$. 
Proposição 3.1. Seja a árvore $T_{!}$. Então, $\mathbb{P}\left(\Lambda_{n}\right) \leq \frac{\left|\partial T_{!, n}\right|}{(n+1) !}=\frac{1}{n+1}$.

Demonstração. Note que, $\Lambda_{n}\left(T_{!}\right)=\bigcup_{v \in \partial T_{!, n}}\{0 \overbrace{\rightarrow}^{\text {c.a }} v\}$. Portanto,

$$
\begin{aligned}
\mathbb{P}\left(\Lambda_{n}\left(T_{!}\right)\right) & =\mathbb{P}\left(\bigcup_{v \in \partial T_{!, n}}\{\mathbf{0} \overbrace{\rightarrow}^{\text {c.a }} v\}\right) \leq \sum_{v \in \partial T_{!, n}} \mathbb{P}(\{\mathbf{0} \overbrace{\rightarrow}^{\text {c.a }} v\}) \\
& =\sum_{v \in \partial T_{!, n}} \frac{1}{(n+1) !}=\frac{\left|\partial T_{!, n}\right|}{(n+1) !}=\frac{n !}{(n+1) !}=\frac{1}{n+1} .
\end{aligned}
$$

Pela observação 3.1, temos

$$
\theta\left(T_{!}\right)=\mathbb{P}\left(\bigcap_{n=1}^{\infty} \Lambda_{n}\right)=\lim _{n \rightarrow \infty} \mathbb{P}\left(\Lambda_{n}\right) \leq \lim _{n \rightarrow \infty} \frac{1}{n+1}=0
$$

Ou seja, $\theta\left(T_{!}\right)=0$. Consequentemente, não há percolação acessível na árvore fatorial.

Consideremos a árvore esfericamente simétrica $T_{\alpha}$ cuja função de crescimento é dada pela função $f(i)=\left\lceil(i+1)^{\alpha}\right\rceil, i \geq 0,\left(\left\lceil(i+1)^{\alpha}\right\rceil=\min \left\{n \in \mathbb{N} ; n \geq(i+1)^{\alpha}\right\}\right)$ onde $\alpha>0$ é uma constante. Pela observação $3.2, \theta(\alpha):=\theta\left(T_{\alpha}\right)$ é uma função não-decrescente em $\alpha$ e o valor crítico

$$
\alpha_{c}:=\inf \{\alpha ; \theta(\alpha)>0\}
$$

está bem definido. O teorema a seguir computa o valor exato onde ocorre a transição de fase para o modelo de percolação acessível em $T_{\alpha}$. 
Teorema 3.3 (Coletti, Gava, R.(2018)). Seja $T_{\alpha}$ tal que $f(i)=\left\lceil(i+1)^{\alpha}\right\rceil, i \geq 0$, onde $\alpha>0$ é uma constante. Então,

$$
\theta\left(T_{\alpha}\right)\left\{\begin{array}{l}
=0, \text { se } \alpha \leq 1 \\
>0, \text { se } \alpha>1
\end{array}\right.
$$

O valor crítico é dado por $\alpha_{c}=1$.

Demonstração. Se $\alpha=1$, então $f(i)=i+1$. Daí, $T_{\alpha}=T_{1}=T_{\text {! }}$ e já vimos que $\theta\left(T_{!}\right)=0$. Agora, se $\alpha<1$, então $f(i)=\left\lceil(i+1)^{\alpha}\right\rceil \leq f(i)=i+1$. Daí, $T_{\alpha} \prec T_{\text {! }}$. Logo, pelo lema 3.1, temos $\theta\left(T_{\alpha}\right) \leq \theta\left(T_{!}\right)=0$. Portanto, $\theta\left(T_{\alpha}\right)=0$ se $\alpha \leq 1$. Para $\alpha>1$, devemos fazer uma comparação com o processo de ramificação com seleção cuja sobrevivência implica em percolação acessível em $T_{\alpha}$. Para tanto, vamos usar um resultado que pode ser encontrado em [7], no teorema de Bertacchi, R. e Zucca, que diz: Suponha que $\sum_{i=0}^{\infty} \frac{1}{m_{i}}<\infty$, e que para algum $C>0$ existe $g: \mathbb{N} \rightarrow[1, \infty)$ tal que $\left(m_{n}{ }^{(2)} /\left(m_{n}{ }^{2}\right) \leq g(n)\right.$, para algum $n$ suficientemente grande e $\lim _{n \rightarrow \infty} \sup (g(n+1) / g(n))<C$. Então, o processo de ramificação com seleção (PRS) sobrevive com probabilidade positiva. Portanto, Se $T$ é esfericamente simétrica com função de crescimento $f$, podemos interpretar $m_{i}=f(i)$, para todo $i \geq 0$ e a condição

$$
\sum_{i=0}^{\infty} \frac{1}{f(i)}<\infty
$$

implica percolação acessível com probabilidade positiva.

Observação 3.3. Para uma prova mais detalhada dessa segunda parte do Teorema 3.3 confira [7]. 


\section{Conclusão}

Neste trabalho fizemos uma estudo detalhado do artigo [1], destacando os principais conceitos relacionados nesse artigo. Buscamos, em todo tempo, deixar o mais claro possível todos os pormenores de cada conceito e resultados que foram abordados, sempre visando uma melhor compreensão da teoria abordada e suas aplicações. Ainda em relação ao artigo [1], discutimos um resultado da literatura que se encontra em [2] que completa o principal resultado obtido no atigo em questão. Sequêncialmente, concluímos com uma discussão sobre os resultados recentes obtidos em percolação acessível tomando por base a teoria desenvolvida em [3]. Discutimos os principais conceitos e os resultados fundamentais que foram obtidos em [3]. Vale ressaltar que em [3] foram estabelecidas novas propriedades do modelo de percolação de acessibilidade em árvores. Contudo, alguns problemas de percolação acessível em árvores permanecem em aberto. Por exemplo, em [3] foi desenvolvida a teoria em cima de árvores esfericamente simétricas e com função de crescimento específica, mas ainda é um problema em aberto obter condições necessárias e suficientes para que exista ou não percolação acessível para uma dada função de crescimento. É fato que, existe uma família inteira de árvores que não valem os resultados obtidos nos artigos $[1,2,3]$. Portanto, é necessário uma busca por novos métodos a serem explorados, que é muito provável o uso do recursos computacionais (simulações numéricas), com o objetivo de resolver estas questões em aberto e que ainda surgirão. 


\section{Referências Bibliográficas}

[1] Nowak, S. and Krug, J., Accessibility percolation on n-trees, EPL 101 (2013), 66004.

[2] Roberts, M. and Zhao, L., Increasing paths in regular trees, Electron. Commun. Probab. 18 (2013), 1-10.

[3] Colleti, C. F., Gava, R. J. and Rodriguez, P. M., On the existence of acessibility in a tree-indexed percolation model, Physica A 492 (2018), 382-388.

[4] Li, L., Phase transition for accessibility percolation on hypercubes, J. Theor. Probab. 31 (2018), 2072-2111.

[5] Cormen, Thomas H., Leiserson, Charles E., Rivest, Ronald L. and Stein, C., Introduction to Algorithms, 3nd ed. Massachusetts Institute of Technology, LondonEngland, 2009.

[9] Berestycki, J., Brunet, É. and Shi, Z., The number of accessible paths in the hypercube, Bernoulli 22 (2016), 653-680.

[7] Bertacchi, D., Rodriguez, P. M. and Zucca, F., Galton-Watson processes in varying environment and accessibility percolation, Braz. J. Probab. Stat. In Press.

[8] Krug, J., Accessibility percolation in random fitness landscapes, arXiv:1903.11913 (2019).

[9] Berestycki, J., Brunet, É. and Shi, Z., Accessibility percolation with backsteps, ALEA, Lat. Am. J. Probab. Math. Stat. 14 (2017), 45-62.

[10] Duque, F., Correa, A. R. and Valencia, L. A., Accessibility percolation with crossing valleys on n-ary trees, J. Stat. Phys. 174 (2019), 1027-1037. 
[11] Hegarty, P. and Martinsson, A., On the existence of accessible paths in various models of fitness landscapes, Ann. Appl. Probab. 24 (2014), 1375-1395.

[12] Martinsson, A., Accessibility percolation and first-passage site percolation on the unoriented binary hypercube, arXiv:1501.02206 (2015). 


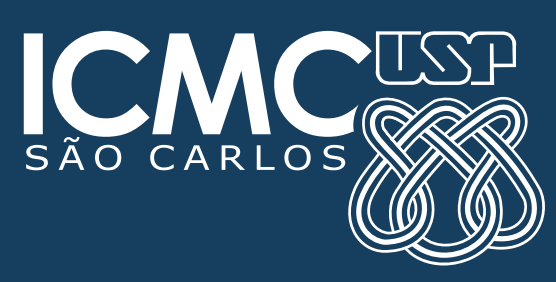

